\title{
GLOBAL SOLUTIONS FOR A NONLINEAR HYPERBOLIC EQUATION WITH BOUNDARY MEMORY SOURCE TERM
}

\author{
FUQIN SUN AND MINGXIN WANG
}

Received 21 January 2005; Accepted 17 August 2005

We study a nonlinear hyperbolic equation with boundary memory source term. By the use of Galerkin procedure, we prove the global existence and the decay property of solution.

Copyright (c) 2006 F. Sun and M. Wang. This is an open access article distributed under the Creative Commons Attribution License, which permits unrestricted use, distribution, and reproduction in any medium, provided the original work is properly cited.

\section{Introduction}

This paper deals with a hyperbolic equation with boundary memory source terms:

$$
\begin{gathered}
\rho(x) u^{\prime \prime}-\Delta u^{\prime}-\Delta u=g(u), \quad x \in \Omega, t>0, \\
u=0, \quad x \in \Gamma_{0}, t>0, \\
\frac{\partial u^{\prime}}{\partial \nu}+\frac{\partial u}{\partial \nu}+f\left(u^{\prime}\right)=\int_{0}^{t} K(t-\tau) h(\tau, u(\tau)) d \tau, \quad x \in \Gamma_{1}, t>0, \\
u(0, x)=u_{0}(x), \quad\left(\sqrt{\rho} u^{\prime}\right)(0, x)=\left(\sqrt{\rho} u_{1}\right)(x), \quad x \in \Omega,
\end{gathered}
$$

where $u=u(t, x), \Omega$ is a bounded domain of $\mathbb{R}^{N}(N \geq 1)$ with sufficiently smooth boundary $\partial \Omega=\Gamma_{0} \cup \Gamma_{1}, \bar{\Gamma}_{0} \cap \bar{\Gamma}_{1}=\varnothing$, where $\Gamma_{0}$ and $\Gamma_{1}$ have positive measures. $u^{\prime}=\partial u / \partial t$, $u^{\prime \prime}=\partial^{2} u / \partial t^{2}$. Equations of type (1.1) are of interest in many applications such as in the theory of electromagnetic materials with memory which obey the Ohm's law. It can also describe the temperature evolution in a rigid conductor with a memory. We refer to $[8,9]$ to see the details. In many works concerned with equations of type (1.1), we cite Aassila et al. [1], where the following wave equation was considered:

$$
\begin{gathered}
u^{\prime \prime}-\Delta u+f_{0}(\nabla u)=0, \quad x \in \Omega, t>0, \\
u=0, \quad x \in \Gamma_{0}, t>0, \\
\frac{\partial u}{\partial v}+g\left(u^{\prime}\right)=\int_{0}^{t} K(t-\tau) h(u(\tau)) d \tau, \quad x \in \Gamma_{1}, t>0, \\
u(0, x)=u_{0}(x), \quad u^{\prime}(0, x)=u_{1}(x), \quad x \in \Omega .
\end{gathered}
$$

Hindawi Publishing Corporation Journal of Inequalities and Applications Volume 2006, Article ID 60734, Pages 1-16 DOI 10.1155/JIA/2006/60734 
Under some conditions on nonlinear terms, they acquired the existence and uniform decay of solutions. Recently, Park and Park [12] generalized problem (1.2) by endowing with some discontinuous and multivalued terms. For more related works, we refer to $[3,4,7,11,13]$ and the references therein. For problem (1.1) without memory source term, we point out the work [6] of Cavalcanti et al., where they investigated the following equation with boundary damping:

$$
\begin{gathered}
\rho(x) u^{\prime \prime}-\Delta u=0, \quad x \in \Omega, t>0, \\
u=0, \quad x \in \Gamma_{0}, t>0, \\
\frac{\partial u}{\partial v}+f\left(u^{\prime}\right)+g(u)=0, \quad x \in \Gamma_{1}, t>0, \\
u(0, x)=u_{0}(x), \quad \sqrt{\rho} u^{\prime}(0, x)=\sqrt{\rho} u_{1}(x), \quad x \in \Omega .
\end{gathered}
$$

Through a partition of boundary $\Gamma$ and Galerkin procedures, they acquired the existence and decay behavior of the solution to problem (1.3). In another work of theirs [5], using similar method, they studied problem (1.3) with $\rho=1$ and the source term $g(u)=|u|^{p} u$ coupled in the first equation. Motivated by the above works, we are devoted to study problem (1.1). By virtue of the potential well method, and through Galerkin procedures, we acquire the global existence and decay property of perturbed energy of solutions of problem (1.1). The organization of this paper is as follows. In Section 2, we make assumptions and introduce a potential well, and then state the main results. In Section 3, making use of Galerkin procedures, we study the existence of solution of problem (1.1). And in the last section, we derive the uniform decay by the perturbed energy method.

\section{Assumptions and main results}

In this section, we first give the notations used throughout this paper:

$$
\begin{array}{cc}
(u, v)=\int_{\Omega} u(x) v(x) d x, & (u, v)_{\Gamma_{1}}=\int_{\Gamma_{1}} u(x) v(x) d \Gamma, \\
\|\cdot\|_{p}=\|\cdot\|_{L^{p}(\Omega)}, & \|\cdot\|=\|\cdot\|_{L^{2}(\Omega),}, \\
\|\cdot\|_{\Gamma_{1}, p}=\|\cdot\|_{L^{p}\left(\Gamma_{1}\right)}, & \|\cdot\|_{\Gamma_{1}}=\|\cdot\|_{L^{2}\left(\Gamma_{1}\right)},
\end{array}
$$

and $r^{\prime}$ denotes the conjugate exponent of $r>1$.

Define

$$
V=\left\{u \in H^{1}(\Omega): u=0 \text { on } \Gamma_{0}\right\}
$$

Since the measure of $\Gamma_{0}$ is positive, Poincarè inequality holds and trace embedding theorem holds (see [2]), we know that $\|\nabla u\|$ is equivalent to the norm on $V$. Let $\mu_{1}$ and $\mu_{2}$ be the optimal constants such that

$$
\|u\| \leq \mu_{1}\|\nabla u\|, \quad\|u\|_{\Gamma_{1}} \leq \mu_{2}\|\nabla u\| \quad \forall u \in V
$$

Now we make the following assumptions. 
$\left(\mathrm{A}_{1}\right) f \in C(\mathbb{R}), f(s) s \geq 0$, and there exist positive constants $k_{1}$ and $k_{2}$ such that

$$
k_{1}|s|^{q-1} \leq|f(s)| \leq k_{2}|s|^{q-1}
$$

where $2<q<\infty$ if $N=1,2 ; 2<q \leq 2(N-1) /(N-2)$ if $N \geq 3$.

$\left(\mathrm{A}_{2}\right) g \in C(\mathbb{R}), g(s) s \geq 0$, and there exists positive constant $k_{3}$ such that

$$
|g(s)| \leq k_{3}|s|^{p}
$$

where $1<p<\infty$ if $N=1,2 ; 1<p \leq N /(N-2)$ if $N \geq 3$.

$\left(\mathrm{A}_{3}\right) K: \mathbb{R}_{+} \rightarrow \mathbb{R}_{+}$is a continuously differentiable function verifying

$$
K^{\prime}(t) \leq-k_{4} K(t) \quad \forall t \geq 0, \quad K(0)>0, \quad 1-\mu_{2}^{2} \int_{0}^{\infty} K(s) d s \triangleq L>0
$$

where $k_{4}>0$.

$\left(\mathrm{A}_{4}\right) h(\tau, s)$ is measurable with $\tau$ and continuous with $s$, and it satisfies

$$
|h(\tau, s)-s| \leq \sqrt{\frac{K(\tau)}{K(0)}}|s| \quad \forall s \in \mathbb{R}, \tau \geq 0
$$

$\left(\mathrm{A}_{5}\right) \rho(x) \geq 0, \rho \neq \equiv$ and $\rho \in L^{\infty}(\Omega)$.

$\left(\mathrm{A}_{6}\right)$ Assume that the initial data

$$
u_{0}, u_{1} \in V \cap H^{3 / 2}(\Omega)
$$

and satisfy the compatibility conditions

$$
\begin{gathered}
-\Delta\left(u_{0}+u_{1}\right)=g\left(u_{0}\right), \quad x \in \Omega, \\
u_{0}=0, \quad u_{1}=0, \quad x \in \Gamma_{0}, \\
\frac{\partial u_{0}}{\partial \nu}+\frac{\partial u_{1}}{\partial \nu}+f\left(u_{1}\right)=0, \quad x \in \Gamma_{1} .
\end{gathered}
$$

Remark 2.1. (i) The assumptions $\left(\mathrm{A}_{3}\right)$ and $\left(\mathrm{A}_{4}\right)$ imply that $h(\tau, s) \approx(1+\sqrt{K(\tau)}) s$.

(ii) Given $u_{1} \in V \cap H^{3 / 2}(\Omega)$, by the assumption $\left(\mathrm{A}_{2}\right)$ and the theory of elliptic problems, we see that problem (2.9) admits a weak solution $u_{0} \in V \cap H^{3 / 2}(\Omega)$.

Let $B_{*}>0$ be the optimal constant such that

$$
\|v\|_{p+1} \leq B_{*}\|\nabla v\| \quad \forall v \in V
$$

where $p$ is the number given in the assumption $\left(\mathrm{A}_{2}\right)$. 
4 Global solutions for a nonlinear hyperbolic equation

If we define

$$
B_{\infty} \triangleq \sup _{v \in V, v \neq 0}\left(\frac{(1 /(p+1))\|v\|_{p+1}^{p+1}}{\|\nabla v\|^{p+1}}\right)
$$

then

$$
B_{\infty} \leq \frac{B_{*}^{p+1}}{p+1}, \quad \frac{1}{p+1}\|v\|_{p+1}^{p+1} \leq B_{\infty}\|\nabla v\|^{p+1} \quad \forall v \in V .
$$

Now for some function $u$, we define

$$
\begin{gathered}
J(u)=\frac{L}{2}\|\nabla u\|^{2}-\frac{k_{3}}{p+1}\|u\|_{p+1}^{p+1}, \\
E(t)=\frac{1}{2}\left\|\sqrt{\rho} u^{\prime}\right\|^{2}+\frac{1}{2}\|\nabla u\|^{2}-\int_{\Omega} G(u) d x-\frac{1}{2}\left(\int_{0}^{t} K(\tau) d \tau\right)\|u(t)\|_{\Gamma_{1}}^{2}+\frac{1}{2}(K \square u)(t),
\end{gathered}
$$

where

$$
G(s)=\int_{0}^{s} g(\eta) d \eta, \quad(K \square u)(t)=\int_{0}^{t} K(t-\tau)\|h(\tau, u(\tau))-u(t)\|_{\Gamma_{1}}^{2} d \tau .
$$

Putting

$$
d \triangleq \inf _{u \in V, u \neq 0}\left\{\sup _{\lambda>0} J(\lambda u)\right\}, \quad H(\lambda) \triangleq \frac{L}{2} \lambda^{2}-k_{3} B_{\infty} \lambda^{p+1}, \quad \lambda>0
$$

We have the following result.

Proposition 2.2. Let the assumptions $\left(A_{2}\right)-\left(A_{4}\right)$ be fulfilled. It holds that

$$
d=\max _{\lambda>0} H(\lambda)=H\left(\lambda_{\infty}\right)=\frac{(p-1) L}{2(p+1)} \lambda_{\infty}^{2}
$$

where $\lambda_{\infty}=\left(L /(p+1) k_{3} B_{\infty}\right)^{1 /(p-1)}$.

If $\|\nabla u\|<\lambda_{\infty}$, then

$$
J(u) \geq 0, \quad\|\nabla u\|^{2} \leq \frac{2(p+1)}{(p-1) L} E(t) .
$$

Proof. From

$$
H^{\prime}(\lambda)=L \lambda-(p+1) k_{3} B_{\infty} \lambda^{p}=\left[L-(p+1) k_{3} B_{\infty} \lambda^{p-1}\right] \lambda
$$


we see that $\lambda_{\infty}=\left[L /\left((p+1) k_{3} B_{\infty}\right)\right]^{1 /(p-1)}$ is the maximum point of $H$. Hence,

$$
\max _{\lambda>0} H(\lambda)=H\left(\lambda_{\infty}\right)=\frac{(p-1) L}{2(p+1)} \lambda_{\infty}^{2}
$$

Note the definition of $B_{\infty}$, by the direct computation, we have

$$
\begin{aligned}
d & =\inf _{u \in V, u \neq 0}\left\{\sup _{\lambda>0} J(\lambda u)\right\} \\
& =\left[\frac{L}{2}\left(\frac{L}{k_{3}}\right)^{2 /(p-1)}-\frac{k_{3}}{p+1}\left(\frac{L}{k_{3}}\right)^{(p+1) /(p-1)}\right] \inf _{u \in V, u \neq 0}\left(\frac{\|\nabla u\|^{p+1}}{\|u\|_{p+1}^{p+1}}\right)^{2 /(p-1)} \\
& =\frac{(p-1) L}{2(p+1)}\left(\frac{L}{(p+1) k_{3} B_{\infty}}\right)^{2 /(p-1)}=\frac{(p-1) L}{2(p+1)} \lambda_{\infty}^{2} .
\end{aligned}
$$

Thus the first conclusion is valid.

If $\|\nabla u\|<\lambda_{\infty}$, then we obtain

$$
\begin{aligned}
E(t) & \geq J(u(t)) \geq \frac{L}{2}\|\nabla u\|^{2}-k_{3} B_{\infty}\|\nabla u\|^{p+1}>\|\nabla u\|^{2}\left(\frac{L}{2}-k_{3} B_{\infty} \lambda_{\infty}^{p-1}\right) \\
& =\|\nabla u\|^{2}\left(\frac{L}{2}-\frac{L}{p+1}\right)=\frac{(p-1) L}{2(p+1)}\|\nabla u\|^{2} .
\end{aligned}
$$

Thus the second conclusion is valid.

Remark 2.3. The number $d$ defined in Proposition 2.2 is the Mountain Pass level related to the elliptic problem

$$
\begin{gathered}
-L \Delta u=k_{3}|u|^{p-1} u, \quad x \in \Omega, \\
u=0, \quad x \in \Gamma_{0}, \\
\frac{\partial u}{\partial v}=0, \quad x \in \Gamma_{1},
\end{gathered}
$$

see [5] or [14]. In fact, $d$ is equal to the number

$$
\inf _{\alpha \in \Lambda} \sup _{t \in[0,1]} J(\alpha(t))
$$

where

$$
\Lambda=\{\alpha \in C([0,1] ; V) ; \alpha(0)=0, J(\alpha(1))<0\}
$$


6 Global solutions for a nonlinear hyperbolic equation

Now we are in a position to state the main results of this paper.

THeOREM 2.4. Let the assumptions $\left(A_{1}\right)-\left(A_{6}\right)$ hold. If in addition, the initial data satisfy

$$
\left\|\nabla u_{0}\right\|<\lambda_{\infty}, \quad E(0)<d
$$

then for any $T>0$, problem (1.1) admits a solution $u \in L^{\infty}(0, T ; V)$ and satisfies $\sqrt{\rho} u^{\prime} \in$ $L^{\infty}\left(0, T ; L^{2}(\Omega)\right), u^{\prime} \in L^{2}(0, T ; V), \rho u^{\prime \prime} \in L^{q^{\prime}}\left(0, T ; L^{2}(\Omega)\right)$.

Theorem 2.5. Let $u$ be the solution obtained in Theorem 2.4. If $q=2$, then the solution $u$ verifies the following decay estimate:

$$
E(t) \leq 3 d \exp \left(-\frac{2}{3} C t\right) \quad \forall t \geq 0
$$

for some positive constant $C$.

\section{Proof of Theorem 2.4}

In this section, we will use Faedo-Galerkin procedure to prove Theorem 2.4.

Step 1. Let $\left\{\omega_{k}\right\}_{k=1}^{\infty}$ be a basis in $V$, which is orthogonal in $L^{2}(\Omega)$. For fixed $n$, let

$$
V_{n}=\left[\omega_{1}, \ldots, \omega_{n}\right]
$$

be the linear span of $\left\{\omega_{k}\right\}_{k=1}^{n}$, and let

$$
\rho_{\varepsilon}=\rho+\varepsilon \quad(\varepsilon>0), \quad u_{\varepsilon n}(t)=\sum_{k=1}^{n} q_{\varepsilon k n}(t) \omega_{k} \in V_{n} .
$$

Consider the Cauchy problem:

$$
\begin{gathered}
\left(\rho_{\varepsilon} u_{\varepsilon n}^{\prime \prime}, \omega\right)+\left(\nabla u_{\varepsilon n}^{\prime}(t), \nabla \omega\right)+\left(\nabla u_{\varepsilon n}(t), \nabla \omega\right)+\left(f\left(u_{\varepsilon n}^{\prime}\right), \omega\right)_{\Gamma_{1}} \\
=\left(g\left(u_{\varepsilon n}\right), \omega\right)+\int_{0}^{t} K(t-\tau)\left(h\left(\tau, u_{\varepsilon n}(\tau)\right), \omega\right)_{\Gamma_{1}} d \tau, \quad \forall \omega \in V_{n}, \\
u_{\varepsilon n}(0)=\sum_{k=1}^{n} q_{\varepsilon k n}(0) \omega_{k} \longrightarrow u_{0} \quad \text { strongly in } V \\
u_{\varepsilon n}^{\prime}(0)=\sum_{k=1}^{n} q_{\varepsilon k n}^{\prime}(0) \omega_{k} \longrightarrow u_{1} \quad \text { strongly in } L^{2}(\Omega) .
\end{gathered}
$$

By the standard method of ordinary differential equations, system (3.3)-(3.4) has a local solution $u_{\varepsilon n}(t)$ on interval $\left(0, t_{\varepsilon n}\right)$ with $q_{\varepsilon k n}(t) \in W^{2,1}\left(0, t_{\varepsilon n}\right)$. The extension of this solution to the whole interval $[0, \infty)$ will be deduced by a series a priori estimates. 
Using the method exploited in the paper [15], we can construct the energy function and the energy identity associated to problem (3.3)-(3.4) as follows:

$$
\begin{gathered}
E_{\varepsilon n}(t)=\frac{1}{2}\left\|\left.\sqrt{\rho_{\varepsilon}} u_{\varepsilon n}^{\prime}\right|^{2}+\frac{1}{2}\right\| \nabla u_{\varepsilon n} \|^{2}-\int_{\Omega} G\left(u_{\varepsilon n}\right) d x \\
\quad-\frac{1}{2}\left(\int_{0}^{t} K(\tau) d \tau\right)\left\|u_{\varepsilon n}(t)\right\|_{\Gamma_{1}}^{2}+\frac{1}{2}\left(K \square u_{\varepsilon n}\right)(t), \\
E_{\varepsilon n}(t)-E_{\varepsilon n}(s)=-\int_{s}^{t} \int_{\Gamma_{1}} f\left(u_{\varepsilon n}^{\prime}(\eta)\right) u_{\varepsilon n}^{\prime}(\eta) d \Gamma d \eta \\
-\int_{s}^{t}\left\|\nabla u_{\varepsilon n}^{\prime}(\eta)\right\|^{2} d \eta+\frac{1}{2} \int_{s}^{t}\left(K^{\prime} \square u_{\varepsilon n}\right)(\eta) d \eta \\
+\frac{K(0)}{2} \int_{s}^{t}\left\|h\left(\eta, u_{\varepsilon n}(\eta)\right)-u_{\varepsilon n}(\eta)\right\|_{\Gamma_{1}}^{2} d \eta-\frac{1}{2} \int_{s}^{t} K(\eta)\left\|u_{\varepsilon n}(\eta)\right\|_{\Gamma_{1}}^{2} d \eta
\end{gathered}
$$

for $0 \leq s \leq t<t_{\varepsilon n}$.

Using the assumption $\left(\mathrm{A}_{4}\right)$, it is easily known that

$$
\frac{K(0)}{2} \int_{s}^{t}\left\|h\left(\eta, u_{\varepsilon n}(\eta)\right)-u_{\varepsilon n}(\eta)\right\|_{\Gamma_{1}}^{2} d \eta-\frac{1}{2} \int_{s}^{t} K(\eta)\left\|u_{\varepsilon n}(\eta)\right\|_{\Gamma_{1}}^{2} d \eta \leq 0
$$

Then using the assumption $\left(\mathrm{A}_{3}\right),(3.7)$ and (3.8) imply that $E_{\varepsilon n}(t)$ is a decreasing function. By the assumption $\left(A_{2}\right)$, we see that

$$
|G(u)| \leq C_{1}|u|^{p+1}
$$

Here and in the sequel $C_{i}, i=1,2, \ldots$, will denote various constants independent of $\varepsilon$ and $n$. Exploiting the continuity of the Nemyskii operator and (3.4), it follows that

$$
\int_{\Omega} G\left(u_{0 \varepsilon n}\right) d x \longrightarrow \int_{\Omega} G\left(u_{0}\right) d x \quad \text { as } n \longrightarrow \infty
$$

Therefore, using (3.4) and (3.5), it entails

$$
E_{\varepsilon n}(0) \longrightarrow E(0) \quad \text { as } n \longrightarrow \infty, \varepsilon \longrightarrow 0 \text {. }
$$

Define

$$
\begin{gathered}
B_{n} \triangleq \sup _{u \in V_{n}, u \neq 0}\left(\frac{(1 /(p+1))\|u\|_{p+1}^{p+1}}{\|\nabla u\|^{p+1}}\right), \quad \lambda_{n} \triangleq\left(\frac{L}{(p+1) k_{3} B_{n}}\right)^{1 /(p-1)}, \\
d_{n} \triangleq \frac{(p-1) L}{2(p+1)} \lambda_{n}^{2} .
\end{gathered}
$$


8 Global solutions for a nonlinear hyperbolic equation

By the assumption $\left(\mathrm{A}_{2}\right)$, it follows that

$$
0<B_{n} \leq B_{n+1} \leq \cdots \leq B_{\infty}, \quad \lambda_{\infty} \leq \cdots \leq \lambda_{n+1} \leq \lambda_{n}, d \leq \cdots \leq d_{n+1} \leq d_{n}, \quad n \geq 1 .
$$

By (2.25), (3.4), (3.5), (3.11), and (3.13), we know that, for sufficiently large $n_{0}$ and sufficiently small $\varepsilon_{0}$,

$$
\left\|\nabla u_{\varepsilon n}(0)\right\|<\lambda_{n}, \quad E_{\varepsilon n}(0)<d_{n}, \quad n \geq n_{0}, \varepsilon \leq \varepsilon_{0} .
$$

From now on, we may assume that $n \geq n_{0}$ and $\varepsilon \leq \varepsilon_{0}$. By (3.6) and the assumptions $\left(\mathrm{A}_{2}\right)$ and $\left(\mathrm{A}_{3}\right)$, we deduce that

$$
E_{\varepsilon n}(t) \geq \frac{L}{2}\left\|\nabla u_{\varepsilon n}(t)\right\|^{2}-k_{3} B_{n}\left\|\nabla u_{\varepsilon n}(t)\right\|^{p+1}=H_{n}\left(\left\|\nabla u_{\varepsilon n}(t)\right\|\right)
$$

where $H_{n}(\lambda)=(L / 2) \lambda^{2}-k_{3} B_{n} \lambda^{p+1}$ has the similar property of the function $H$ defined in Proposition 2.2. It is easy to verify that $H_{n}$ is increasing for $0<\lambda<\lambda_{n}$ and decreasing for $\lambda>\lambda_{n}, H_{n}\left(\lambda_{n}\right)=d_{n}$, and $H_{n}(\lambda) \rightarrow-\infty$ as $\lambda \rightarrow+\infty$. Since $E_{\varepsilon n}(0)<d_{n}$, there exist $\lambda_{n}^{1}<\lambda_{n}<$ $\lambda_{n}^{2}$ such that $H_{n}\left(\lambda_{n}^{1}\right)=H_{n}\left(\lambda_{n}^{2}\right)=E_{\varepsilon n}(0)$. From (3.7) and (3.8), we have

$$
E_{\varepsilon n}(t) \leq E_{\varepsilon n}(0) \quad \forall t \in\left[0, t_{\varepsilon n}\right) .
$$

Denote $\lambda_{n}^{0}=\left\|\nabla u_{\varepsilon n}(0)\right\|$, so $\lambda_{n}^{0}<\lambda_{n}$. By (3.15), we have $H_{n}\left(\lambda_{n}^{0}\right) \leq E_{\varepsilon n}(0)$, thus $\lambda_{n}^{0}<\lambda_{n}^{1}$.

We claim that $\left\|\nabla u_{\varepsilon n}(t)\right\| \leq \lambda_{n}^{1}$ for all $t \in\left[0, t_{\varepsilon n}\right)$. Suppose, by contradiction, that $\left\|\nabla u_{\varepsilon n}\left(t_{0}\right)\right\|>\lambda_{n}^{1}$ for some $t_{0} \in\left(0, t_{\varepsilon n}\right)$. From the continuity of $\left\|\nabla u_{\varepsilon n}(\cdot)\right\|$, we can suppose that $\left\|\nabla u_{\varepsilon n}\left(t_{0}\right)\right\|<\lambda_{n}$. Then by (3.15), $E_{\varepsilon n}\left(t_{0}\right) \geq H_{n}\left(\left\|\nabla u_{\varepsilon n}\left(t_{0}\right)\right\|\right)>H_{n}\left(\lambda_{n}^{1}\right)=E_{\varepsilon n}(0)$, which contradicts (3.16). From (3.14) and (3.16), it yields $E_{\varepsilon n}(t)<d_{n}$ for $t \in\left[0, t_{\varepsilon n}\right)$. Then using (3.13), one gets

$$
\left\|\nabla u_{\varepsilon n}(t)\right\| \leq \lambda_{1}, \quad E_{\varepsilon n}(t)<d_{1}
$$

for $t \in\left[0, t_{\varepsilon n}\right)$. By (3.17), the assumption $\left(\mathrm{A}_{2}\right)$, and the Sobolev embedding theorem, we deduce that

$$
\int_{\Omega} G\left(u_{\varepsilon n}\right) d x \leq \frac{k_{3}}{p+1}\left\|u_{\varepsilon n}\right\|_{p+1}^{p+1} \leq C_{2}
$$

Therefore, from (3.6), (3.17), and (3.18), it follows that

$$
\left\|\sqrt{\rho_{\varepsilon}} u_{\varepsilon n}^{\prime}(t)\right\| \leq C_{3} \text {. }
$$

Estimates (3.17) and (3.19) imply that $t_{\varepsilon n}=\infty$. 
For any $T>0$ and for all $t \in[0, T]$, by the assumptions $\left(A_{1}\right),\left(A_{3}\right)$, and $\left(A_{4}\right)$, we get from (3.7), (3.14), and (3.16) that

$$
\begin{gathered}
\int_{0}^{t}\left\|\nabla u_{\varepsilon n}^{\prime}(\tau)\right\|^{2} d \tau \leq C_{4}, \\
\int_{0}^{t}\left\|u_{\varepsilon n}^{\prime}(\tau)\right\|_{\Gamma_{1}, q}^{q} d \tau \leq k_{1}^{-1} \int_{0}^{t}\left(f\left(u_{\varepsilon n}^{\prime}(\tau)\right), u_{\varepsilon n}^{\prime}(\tau)\right)_{\Gamma_{1}} d \tau \leq C_{5} .
\end{gathered}
$$

Then using the assumptions $\left(\mathrm{A}_{1}\right)-\left(\mathrm{A}_{2}\right)$, the Sobolev embedding theorem, (3.13), and (3.20), we derive that, for all $t \in[0, T]$,

$$
\int_{0}^{t}\left\|f\left(u_{\varepsilon n}^{\prime}\right)\right\|_{\Gamma_{1}, q^{\prime}}^{q^{\prime}} d \tau \leq C_{6}, \quad\left\|g\left(u_{\varepsilon n}\right)\right\|_{(p+1)^{\prime}}^{(p+1)^{\prime}} \leq C_{7}
$$

Similarly, by the assumptions $\left(\mathrm{A}_{3}\right)-\left(\mathrm{A}_{4}\right)$ and the Sobolev embedding theorem, it leads to

$$
\int_{0}^{t}\left\|h\left(\tau, u_{\varepsilon n}(\tau)\right)\right\|_{\Gamma_{1}} d \tau \leq C_{8}, \quad\left\|\int_{0}^{t} K(t-\tau) h\left(\tau, u_{\varepsilon n}(\tau)\right) d \tau\right\|_{\Gamma_{1}} \leq C_{9} \quad \forall t \in[0, T] .
$$

Replacing $\omega$ in (3.3) with $v \in V$, and exploiting the Hölder inequality, the Sobolev embedding theorem, (3.17), (3.21), and (3.22), it entails

$$
\begin{aligned}
\left|\left(\rho_{\varepsilon} u_{\varepsilon n}^{\prime \prime}, v\right)\right| \leq C_{10}( & \left\|\nabla u_{\varepsilon n}^{\prime}\right\|+\left\|\nabla u_{\varepsilon n}\right\|+\left\|f\left(u_{\varepsilon n}^{\prime}\right)\right\|_{\Gamma_{1}, q^{\prime}}+\left\|g\left(u_{\varepsilon n}\right)\right\|_{(p+1)^{\prime}} \\
& \left.+\left\|\int_{0}^{t} K(t-\tau) h\left(\tau, u_{\varepsilon n}(\tau)\right) d \tau\right\|_{\Gamma_{1}}\right)\|\nabla v\|, \\
\left\|\rho_{\varepsilon} u_{\varepsilon n}^{\prime \prime}\right\| & \leq C_{11}\left(1+\left\|\nabla u_{\varepsilon n}^{\prime}\right\|+\left\|f\left(u_{\varepsilon n}^{\prime}\right)\right\|_{\Gamma_{1}, q^{\prime}}\right)
\end{aligned}
$$

for all $t \in[0, T]$.

Integrating the above inequality over $[0, t]$, using (3.20)-(3.21) and the Hölder inequality, we get

$$
\int_{0}^{t}\left\|\rho_{\varepsilon} u_{\varepsilon n}^{\prime \prime}\right\|^{q^{\prime}} d \tau \leq C_{12} \int_{0}^{t}\left(1+\left\|\nabla u_{\varepsilon n}^{\prime}\right\|^{q^{\prime}}+\left\|f\left(u_{\varepsilon n}^{\prime}\right)\right\|_{\Gamma_{1}, q^{\prime}}^{q^{\prime}}\right) d \tau \leq C_{13}
$$


10 Global solutions for a nonlinear hyperbolic equation

Step 2. The limiting process. From the estimates (3.17), (3.19)-(3.24), using the standard arguments, it yields that, up to a subsequence, as $n \rightarrow \infty$,

$$
\begin{gathered}
u_{\varepsilon n} \longrightarrow u_{\varepsilon} \quad \text { weakly* in } L^{\infty}(0, T ; V), \\
\sqrt{\rho_{\varepsilon}} u_{\varepsilon n}^{\prime} \longrightarrow \sqrt{\rho_{\varepsilon}} u_{\varepsilon}^{\prime} \quad \text { weakly }{ }^{*} \text { in } L^{\infty}\left(0, T ; L^{2}(\Omega)\right), \\
u_{\varepsilon n}^{\prime} \longrightarrow u_{\varepsilon}^{\prime} \quad \text { weakly in } L^{2}(0, T ; V), \\
f\left(u_{\varepsilon n}^{\prime}\right) \longrightarrow \gamma \quad \text { weakly* in } L^{q^{\prime}}\left((0, T) \times \Gamma_{1}\right), \\
g\left(u_{\varepsilon n}\right) \longrightarrow \chi \quad \text { weakly* in } L^{\infty}\left(0, T ; L^{(p+1)^{\prime}}(\Omega)\right), \\
h\left(t, u_{\varepsilon n}\right) \longrightarrow \xi \quad \text { weakly in } L^{2}\left(0, T ; L^{2}(\Omega)\right), \\
\rho_{\varepsilon} u_{\varepsilon n}^{\prime \prime} \longrightarrow \rho_{\varepsilon} u_{\varepsilon}^{\prime \prime} \quad \text { weakly* in } L^{q^{\prime}}\left(0, T ; L^{2}(\Omega)\right) .
\end{gathered}
$$

Since $V \hookrightarrow L^{2}(\Omega)$ and $V \hookrightarrow L^{2}\left(\Gamma_{1}\right)$, then by the Aubin-Lions compactness lemma [10, Theorem 5.1], we get from (3.25) and (3.26) that, as $n \rightarrow \infty$,

$$
\begin{gathered}
u_{\varepsilon n} \longrightarrow u_{\varepsilon} \quad \text { strongly in } L^{2}\left(0, T ; L^{2}(\Omega)\right) \text { and a.e. on }(0, T) \times \Omega, \\
u_{\varepsilon n} \longrightarrow u_{\varepsilon} \quad \text { strongly in } L^{2}\left(0, T ; L^{2}\left(\Gamma_{1}\right)\right) \text { and a.e. on }(0, T) \times \Gamma_{1}, \\
u_{\varepsilon n}^{\prime} \longrightarrow u_{\varepsilon}^{\prime} \quad \text { strongly in } L^{2}\left(0, T ; L^{2}(\Omega)\right) \text { and a.e. on }(0, T) \times \Omega, \\
u_{\varepsilon n}^{\prime} \longrightarrow u_{\varepsilon}^{\prime} \quad \text { strongly in } L^{2}\left(0, T ; L^{2}\left(\Gamma_{1}\right)\right) \text { and a.e. on }(0, T) \times \Gamma_{1},
\end{gathered}
$$

From (3.25), (3.26), and (3.30), we acquire, as $n \rightarrow \infty$,

$$
\begin{gathered}
\left(u_{\varepsilon n}, \omega\right) \longrightarrow\left(u_{\varepsilon}, \omega\right) \quad \text { weakly in } L^{2}[0, T], \\
\left(u_{\varepsilon n}^{\prime}, \omega\right) \longrightarrow\left(u_{\varepsilon}^{\prime}, \omega\right) \quad \text { weakly in } L^{2}[0, T], \\
\left(\rho_{\varepsilon} u_{\varepsilon n}^{\prime \prime}, \omega\right) \longrightarrow\left(\rho_{\varepsilon} u_{\varepsilon}^{\prime \prime}, \omega\right) \quad \text { weakly in } L^{q^{\prime}}[0, T] .
\end{gathered}
$$

Note that $W^{1,2}[0, T] \hookrightarrow C[0, T]$ and $W^{1, q^{\prime}} \hookrightarrow C[0, T]$, from $(3.35)$, we get that

$$
\left(u_{\varepsilon n}(0), \omega\right) \longrightarrow\left(u_{\varepsilon}(0), \omega\right),\left(u_{\varepsilon n}^{\prime}(0), \omega\right) \longrightarrow\left(u_{\varepsilon}^{\prime}(0), \omega\right),
$$

and hence

$$
u_{\varepsilon}(0)=u_{0} \quad \text { in } V, \quad u_{\varepsilon}^{\prime}(0)=u_{1} \quad \text { in } L^{2}(\Omega) .
$$

Now letting $n \rightarrow \infty$ in (3.3) and using (3.25)-(3.37), we acquire

$$
\begin{gathered}
\int_{0}^{T}\left[\left(\rho_{\varepsilon} u_{\varepsilon}^{\prime \prime}, v\right)+\left(\nabla u_{\varepsilon}^{\prime}, \nabla v\right)+\left(\nabla u_{\varepsilon}, \nabla v\right)+(\gamma, v)_{\Gamma_{1}}-(\chi, v)\right] d t \\
=\int_{0}^{T} \int_{0}^{t} K(t-\tau)(\xi, v)_{\Gamma_{1}} d \tau d t
\end{gathered}
$$

for any $v \in V$. 
Step 3. We first prove $\gamma=f\left(u_{\varepsilon}^{\prime}\right)$. By (3.33), the continuity of $f(s)$, we see that $f\left(u_{\varepsilon n}^{\prime}\right) \rightarrow$ $f\left(u_{\varepsilon}^{\prime}\right)$ and a.e. on $(0, T) \times \Gamma_{1}$. Thus by use of (3.21), and of Lions' [10, Lemma 1.3], we have $f\left(u_{\varepsilon n}^{\prime}\right) \rightarrow f\left(u_{\varepsilon}^{\prime}\right)$ weakly in $L^{q^{\prime}}\left((0, T) \times \Gamma_{1}\right)$. Then (3.27) and the uniqueness of weak* limit give $\gamma=f\left(u_{\varepsilon}^{\prime}\right)$ in $L^{q^{\prime}}\left((0, T) \times \Gamma_{1}\right)$. By analogous analysis, one can get $\xi=h\left(t, u_{\varepsilon}(t)\right)$ in $L^{2}\left((0, T) \times \Gamma_{1}\right)$.

Below we show $\chi=g\left(u_{\varepsilon}\right)$ along the line of the paper [16]. By (3.31) and the continuity of $g(s)$, we have $g\left(u_{\varepsilon n}\right) \rightarrow g\left(u_{\varepsilon}\right)$ a.e. on $Q_{T}=(0, T) \times \Omega$. Therefore, exploiting Lusin and Egoroff's theorem, for any $\delta>0$, there exists a measurable set $Q \subset Q_{T}$ such that $|Q|<\delta$, and $g\left(u_{\varepsilon n}\right) \rightarrow g\left(u_{\varepsilon}\right)$ uniformly on $Q_{T} \backslash Q$ as $n \rightarrow \infty$. By the Sobolev embedding theorem, we know from (3.25) that $u_{\varepsilon} \in L^{p+1}\left(Q_{T}\right)$. And hence the assumption $\left(\mathrm{A}_{2}\right)$ implies that $g\left(u_{\varepsilon}\right) \in L^{(p+1)^{\prime}}\left(Q_{T}\right)$. For any $\hat{p}>p$, by the use of (3.28), we get

$$
\begin{aligned}
\left\|g\left(u_{\varepsilon n}\right)-g\left(u_{\varepsilon}\right)\right\|_{L^{(\hat{p}+1)^{\prime}}(Q)} & \leq\left\|g\left(u_{\varepsilon n}\right)-g\left(u_{\varepsilon}\right)\right\|_{L^{(p+1)^{\prime}}(Q)} \delta^{(\hat{p}-p) /(\hat{p}+1)(p+1)} \\
& \leq C_{14} \delta^{(\hat{p}-p) /(\hat{p}+1)(p+1)} .
\end{aligned}
$$

So, as $n \rightarrow \infty$,

$$
\begin{aligned}
& \left\|g\left(u_{\varepsilon n}\right)-g\left(u_{\varepsilon}\right)\right\|_{L^{(\hat{p}+1)^{\prime}}\left(Q_{T}\right)} \\
& \quad \leq\left\|g\left(u_{\varepsilon n}\right)-g\left(u_{\varepsilon}\right)\right\|_{L^{(\hat{p}+1)^{\prime}}(Q)}+\left\|g\left(u_{\varepsilon n}\right)-g\left(u_{\varepsilon}\right)\right\|_{L^{(\hat{p}+1)^{\prime}}\left(Q_{T} \backslash Q\right)} \\
& \quad \leq C_{15} \delta^{(\hat{p}-p) /(\hat{p}+1)(p+1)} .
\end{aligned}
$$

By the arbitrariness of $\delta$, we get that, as $n \rightarrow \infty$,

$$
g\left(u_{\varepsilon n}\right) \longrightarrow g\left(u_{\varepsilon}\right) \quad \text { strongly in } L^{(\hat{p}+1)^{\prime}}\left(Q_{T}\right)
$$

Using (3.28), (3.41), and the uniqueness of weak* limit, we acquire that $\chi=g\left(u_{\varepsilon}\right)$ in $L^{(\hat{p}+1)^{\prime}}\left(Q_{T}\right)$.

Because $C_{0}^{\infty}\left(Q_{T}\right)$ is dense in $L^{p+1}\left(Q_{T}\right)$, for any $\phi \in L^{p+1}\left(Q_{T}\right)$, we can choose a sequence $\left\{\phi_{n}\right\}, \phi_{n}$ in $C_{0}^{\infty}\left(Q_{T}\right)(n=1,2, \ldots)$, such that $\phi_{n} \rightarrow \phi$ strongly in $L^{p+1}\left(Q_{T}\right)$, and

$$
\left|\int_{0}^{T}\left(g\left(u_{\varepsilon}\right)-\chi, \phi_{n}-\phi\right) d t\right| \leq\left\|g\left(u_{\varepsilon}\right)-\chi\right\|_{L^{(p+1)^{\prime}}\left(Q_{T}\right)}\left\|\phi_{n}-\phi\right\|_{L^{p+1}\left(Q_{T}\right)} \longrightarrow 0
$$

as $n \rightarrow \infty$. From (3.42), it follows that

$$
\begin{gathered}
\int_{0}^{T}\left(g\left(u_{\varepsilon}\right)-\chi, \phi\right) d t=\lim _{n \rightarrow \infty} \int_{0}^{T}\left(g\left(u_{\varepsilon}\right)-\chi, \phi_{n}\right) d t \longrightarrow 0, \\
g\left(u_{\varepsilon}\right)=\chi \quad \text { in } L^{(p+1)^{\prime}}\left(Q_{T}\right) .
\end{gathered}
$$

Note that all the estimates above are independent of $\varepsilon$, then using the similar arguments as above and letting $\varepsilon \rightarrow 0$, there exists a limit function $u$ of $u_{\varepsilon}$ being the solution of problem (1.1). The proof of Theorem 2.4 is completed. 
12 Global solutions for a nonlinear hyperbolic equation

\section{Proof of Theorem 2.5}

In order to derive the decay property of the solution given by Theorem 2.4 , we divide the proof into two cases.

Case 1. Suppose that

$$
\int_{0}^{t}\left(\left\|\sqrt{\rho} u^{\prime}(\tau)\right\|^{2}+\|\nabla u(\tau)\|^{2}\right) d \tau \leq E(t)
$$

for all $t \geq 0$. In this situation, we first prove two lemmas, then based on them, we complete the proof.

Define

$$
m(t)=\left(\rho u^{\prime}(t), u(t)\right)-k \int_{0}^{t}\left[\left\|\sqrt{\rho} u^{\prime}(\tau)\right\|^{2}+\|\nabla u(\tau)\|^{2}\right] d \tau, \quad E^{\sigma}(t)=E(t)+\sigma m(t),
$$

where $k=\mu_{2}^{2}\|K\|_{L^{1}(0, \infty)}+(p+1) L / 2$.

Lemma 4.1. There exists $K_{1}>0$ such that for each $\sigma>0$,

$$
\left|E^{\sigma}(t)-E(t)\right| \leq \sigma K_{1} E(t) \quad \forall t \geq 0 .
$$

Proof. By the definition of $B_{n}$ and $B_{\infty}$, it is easy to get $\lim _{n \rightarrow \infty} B_{n}=B_{\infty}$, and so

$$
\lim _{n \rightarrow \infty} \lambda_{n}=\lambda_{\infty}, \quad \lim _{n \rightarrow \infty} d_{n}=d_{\infty} .
$$

Since $\left\|\nabla u_{\varepsilon n}(t)\right\| \leq \lambda_{n}$ and $E_{\varepsilon n}(t) \leq d_{n}$, by letting $n \rightarrow \infty$ and $\varepsilon \rightarrow 0$, we know that $\|\nabla u(t)\| \leq \lambda_{\infty}$ and $E(t) \leq d_{\infty}$. Therefore, by the conclusion of Proposition 2.2, we know that

$$
\begin{gathered}
\|\nabla u(t)\|^{2} \leq \frac{2(p+1)}{(p-1) L} E(t), \\
\frac{1}{2}\|\nabla u(t)\|^{2}-\int_{\Omega} G(u(t)) d x \geq J(u(t)) \geq 0 .
\end{gathered}
$$

Then by the assumptions $\left(A_{3}\right)-\left(A_{4}\right)$ and the second inequality of $(2.3)$, it is easy to show

$$
E(t) \geq \frac{1}{2}\left\|\sqrt{\rho} u^{\prime}(t)\right\|^{2}+\frac{L}{2}\|\nabla u(t)\|^{2}-\int_{\Omega} G(u(t)) d x .
$$

Applying the Hölder inequality, the Young inequality, (2.3), and (4.5)-(4.7), we have

$$
\begin{aligned}
\left|\left(\rho u^{\prime}(t), u(t)\right)\right| & \leq\|\sqrt{\rho}\|_{\infty}\left\|\sqrt{\rho} u^{\prime}(t) \mid\right\| u(t) \| \\
& \leq \frac{\|\sqrt{\rho}\|_{\infty}^{2}}{2}\left\|\sqrt{\rho} u^{\prime}(t)\right\|^{2}+\frac{\mu_{1}^{2}}{2}\|\nabla u(t)\|^{2} \\
& \leq\left(\|\sqrt{\rho}\|_{\infty}^{2}+\frac{p+1}{p-1} L^{-1} \mu_{1}^{2}\right) E(t) .
\end{aligned}
$$

Take $K_{1}=k+\|\sqrt{\rho}\|_{\infty}^{2}+((p+1) /(p-1)) L^{-1} \mu_{1}^{2}$, we conclude the result. 
Lemma 4.2. There exist $K_{2}>0$ and $\sigma_{1}>0$ such that

$$
\left(E^{\sigma}\right)^{\prime}(t) \leq-\sigma K_{2} E(t) \quad \forall t \geq 0, \forall \sigma \in\left(0, \sigma_{1}\right] .
$$

Proof. Using problem (1.1), we get that

$$
\begin{aligned}
m^{\prime}(t)= & \left(\rho u^{\prime \prime}(t), u(t)\right)+\left(\rho u^{\prime}(t), u^{\prime}(t)\right)-k\left[\left\|\sqrt{\rho} u^{\prime}(t)\right\|^{2}+\|\nabla u(t)\|^{2}\right] \\
= & -\left(\nabla u^{\prime}(t), \nabla u(t)\right)-\|\nabla u(t)\|^{2}-\left(f\left(u^{\prime}(t)\right), u(t)\right)_{\Gamma_{1}}+(g(u(t)), u(t)) \\
& +\int_{0}^{t} K(t-\tau)(h(\tau, u(\tau)), u(t))_{\Gamma_{1}} d \tau+\left(\rho u^{\prime}(t), u^{\prime}(t)\right)-k\left[\left\|\sqrt{\rho} u^{\prime}(t)\right\|^{2}+\|\nabla u(t)\|^{2}\right] \\
\leq & -E(t)+\frac{3}{2}\|\rho\|_{\infty}\left\|u^{\prime}(t)\right\|^{2}-\left(\nabla u^{\prime}(t), \nabla u(t)\right)-\left(\frac{1}{2}+k\right)\|\nabla u(t)\|^{2}-\int_{\Omega} G(u(t)) d x \\
& -\frac{1}{2}\left(\int_{0}^{t} K(\tau) d \tau\right)\|u\|_{\Gamma_{1}}^{2}+\frac{1}{2}(K \square u)(t)-\left(f\left(u^{\prime}(t)\right), u(t)\right) \\
& +(g(u(t)), u(t))+\int_{0}^{t} K(t-\tau)(h(\tau, u(\tau)), u(t))_{\Gamma_{1}} d \tau .
\end{aligned}
$$

Exploiting assumptions $\left(A_{3}\right),\left(A_{4}\right)$, the Hölder inequality, the Young inequality, and (2.3), we have

$$
\begin{aligned}
& \left|\int_{0}^{t} K(t-\tau)(h(\tau, u(\tau)), u(t))_{\Gamma_{1}} d \tau\right| \\
& \quad \leq \mu_{2}^{2}\|K\|_{L^{1}(0, \infty)}\|\nabla u(t)\|^{2}+\frac{1}{4} \int_{0}^{t} K(t-\tau)\|h(\tau, u(\tau))\|_{\Gamma_{1}}^{2} d \tau \\
& \quad \leq \mu_{2}^{2}\|K\|_{L^{1}(0, \infty)}|| \nabla u(t)\left\|^{2}+\frac{1}{2}(K \square u)(t)+\frac{1}{2}\left(\int_{0}^{t} K(\tau) d \tau\right)\right\| u(t) \|_{\Gamma_{1}}^{2}, \\
& \left|-\left(\nabla u^{\prime}(t), \nabla u(t)\right)\right| \leq \frac{1}{4}\|\nabla u(t)\|^{2}+\left\|\nabla u^{\prime}(t)\right\|^{2} .
\end{aligned}
$$

By the assumption $\left(A_{2}\right)$ and noting (4.6), we get

$$
|(g(u(t)), u(t))| \leq k_{3}\|u(t)\|_{p+1}^{p+1} \leq \frac{(p+1) L}{2}\|\nabla u(t)\|^{2}
$$

Applying the assumption $\left(\mathrm{A}_{1}\right)$ with $q=2$, the Hölder inequality, the trace embedding theorem, and the Young inequality, we have

$$
\left|-\left(f\left(u^{\prime}(t)\right), u(t)\right)_{\Gamma_{1}}\right| \leq k_{2}\left\|u^{\prime}(t)\right\|_{\Gamma_{1}}\|u(t)\|_{\Gamma_{1}} \leq k_{2}^{2} \mu_{2}^{4}|| \nabla u^{\prime}(t)\left\|^{2}+\frac{1}{4}\right\| \nabla u(t) \|^{2} .
$$

Combining (4.5), (4.10)-(4.14), and the assumptions $\left(\mathrm{A}_{2}\right)$ and $\left(\mathrm{A}_{4}\right)$, we get

$$
m^{\prime}(t) \leq-E(t)\left(\frac{3}{2}\|\rho\|_{\infty} \mu_{1}^{2}+k_{2}^{2} \mu_{2}^{4}+1\right)\left\|\nabla u^{\prime}(t)\right\|^{2}+(K \square u)(t) .
$$


14 Global solutions for a nonlinear hyperbolic equation

Moreover, by the assumptions $\left(A_{1}\right),\left(A_{3}\right)$, and $\left(A_{4}\right)$, we get from (3.7) and (3.8) that

$$
\begin{aligned}
E^{\prime}(t) & \leq-\left\|\nabla u^{\prime}(t)\right\|^{2}+\frac{1}{2}\left(K^{\prime} \square u\right)(t) \\
& \leq-\left\|\nabla u^{\prime}(t)\right\|^{2}-\frac{k_{4}}{2}(K \square u)(t) .
\end{aligned}
$$

Combining (4.15) and (4.16), we get

$$
\begin{aligned}
\left(E^{\sigma}\right)^{\prime}(t)= & E^{\prime}(t)+\sigma m^{\prime}(t) \leq-\sigma E(t)-\left(\frac{k_{4}}{2}-\sigma\right)(K \square u)(t) \\
& -\left[1-\sigma\left(\frac{3}{2}\|\rho\|_{\infty} \mu_{1}^{2}+k_{2}^{2} \mu_{2}^{4}+1\right)\right]\left\|\nabla u^{\prime}(t)\right\|^{2}
\end{aligned}
$$

Now we choose $\sigma$ sufficiently small such that

$$
\frac{k_{4}}{2}-\sigma>0, \quad 1-\sigma\left(\frac{3}{2}\|\rho\|_{\infty} \mu_{1}^{2}+k_{2}^{2} \mu_{2}^{4}+1\right)>0 .
$$

Then take $K_{2}=\sigma$ in (4.17) to complete the proof.

Completion of the proof of Case 1 . Let $\sigma_{0}=\min \left\{1 /\left(2 K_{1}\right), \sigma_{1}\right\}$. Then for $\sigma \in\left(0, \sigma_{0}\right]$, we know from Lemma 4.1 that

$$
\frac{1}{2} E(t) \leq E^{\sigma}(t) \leq \frac{3}{2} E(t)
$$

Set $K_{3}=\sigma K_{2}=\sigma^{2}$, by Lemma 4.2 and (4.19), it yields

$$
\left(E^{\sigma}\right)^{\prime}(t) \leq-K_{3} E(t) \leq-\frac{2}{3} K_{3} E^{\sigma}(t)
$$

Hence

$$
\frac{d}{d t}\left[E^{\sigma}(t) \exp \left(\frac{2}{3} K_{3} t\right)\right] \leq 0 \quad \forall t \geq 0 .
$$

Integrating (4.21) and using (4.19), we have

$$
E(t) \leq 3 E(0) \exp \left(-\frac{2}{3} K_{3} t\right) \quad \forall t \geq 0 .
$$

The proof of Case 1 is completed.

Case 2. Suppose that there exists a $t_{0} \geq 0$ such that

$$
\int_{0}^{t_{0}}\left(\left\|\sqrt{\rho} u^{\prime}(\tau)\right\|^{2}+\|\nabla u(\tau)\|^{2}\right) d \tau>E\left(t_{0}\right)
$$

Without loss of generality, we suppose that $t_{0}>0$ and is the first one such that the above 
inequality holds. This fells out that

$$
\int_{0}^{t_{0}}\left(\left\|\sqrt{\rho} u^{\prime}(\tau)\right\|^{2}+\|\nabla u(\tau)\|^{2}\right) d \tau \leq E(t) \quad \forall t \in\left[0, t_{0}\right)
$$

Then along the line of proofs for Case 1 , we deduce that (4.22) holds for each $t \in\left[0, t_{0}\right)$.

On the other hand, by (4.5)-(4.6) and the nonincreasing property of $E(t)$, we easily get that for all $t \geq t_{0}$,

$$
\begin{aligned}
\left\|\sqrt{\rho} u^{\prime}(t)\right\|^{2}+\|\nabla u(t)\|^{2} & \leq C E(t) \leq C E\left(t_{0}\right) \\
& \leq C \int_{0}^{t_{0}}\left[\left\|\sqrt{\rho} u^{\prime}(\tau)\right\|^{2}+\|\nabla u(\tau)\|^{2}\right] d \tau \\
& \leq C \int_{0}^{t}\left[\left\|\sqrt{\rho} u^{\prime}(\tau)\right\|^{2}+\|\nabla u(\tau)\|^{2}\right] d \tau,
\end{aligned}
$$

where $C$ is a positive constant. Then by the Gronwall inequality and noting that $\rho \neq 0$, we infer from (4.25) that $u=0$ as $t \geq t_{0}$. Therefore, the decay property of $u$ is trivial for $t \geq t_{0}$. Case 2 is proved.

Combining Case 1 and Case 2, we complete the proof Theorem 2.5.

\section{Acknowledgments}

This work was supported by the National Natural Science Foundation of China 10471022, and the Ministry of Education of China, Science and Technology Major Projects, Grant 104090. The authors thank the referee for his/her valuable comments and suggestions.

\section{References}

[1] M. Aassila, M. M. Cavalcanti, and V. N. Domingos Cavalcanti, Existence and uniform decay of the wave equation with nonlinear boundary damping and boundary memory source term, Calculus of Variations and Partial Differential Equations 15 (2002), no. 2, 155-180.

[2] R. A. Adams, Sobolev Spaces, Academic Press, New York, 1975.

[3] J. J. Bae, Uniform decay for the unilateral problem associated to the Kirchhoff type wave equations with nonlinear boundary damping, Applied Mathematics and Computation 156 (2004), no. 1, $41-57$.

[4] M. M. Cavalcanti, Existence and uniform decay for the Euler-Bernoulli viscoelastic equation with nonlocal boundary dissipation, Discrete and Continuous Dynamical Systems 8 (2002), no. 3, 675-695.

[5] M. M. Cavalcanti, V. N. Domingos Cavalcanti, and P. Martinez, Existence and decay rate estimates for the wave equation with nonlinear boundary damping and source term, Journal of Differential Equations 203 (2004), no. 1, 119-158.

[6] M. M. Cavalcanti, V. N. Domingos Cavalcanti, and J. A. Soriano, On existence and asymptotic stability of solutions of the degenerate wave equation with nonlinear boundary conditions, Journal of Mathematical Analysis and Applications 281 (2003), no. 1, 108-124.

[7] C. Giorgi and V. Pata, Asymptotic behavior of a nonlinear hyperbolic heat equation with memory, Nonlinear Differential Equations and Applications 8 (2001), no. 2, 157-171. 
[8] D. Graffi, Qualche problema di elettromagnetismo, Trends in Applications of Pure Mathematics to Mechanics (Conf., Univ. Lecce, Lecce, 1975) (G. Fichera, ed.), Monographs and Studies in Math., vol. 2, Pitman, London, 1976, pp. 129-144.

[9] M. E. Gurtin and A. C. Pipkin, A general theory of heat conduction with finite wave speeds, Archive for Rational Mechanics and Analysis 31 (1968), 113-126.

[10] J.-L. Lions, Quelques méthodes de résolution des problèmes aux limites non linéaires, Dunod; Gauthier-Villars, Paris, 1969.

[11] J. Y. Park and J. J. Bae, On coupled wave equation of Kirchhoff type with nonlinear boundary damping and memory term, Applied Mathematics and Computation 129 (2002), no. 1, 87-105.

[12] J. Y. Park and S. H. Park, On solutions for a hyperbolic system with differential inclusion and memory source term on the boundary, Nonlinear Analysis. Theory, Methods \& Applications. Series A: Theory and Methods 57 (2004), no. 3, 459-472.

[13] V. Pata, Attractors for a damped wave equation on $\mathbb{R}^{3}$ with linear memory, Mathematical Methods in the Applied Sciences 23 (2000), no. 7, 633-653.

[14] E. Vitillaro, A potential well theory for the wave equation with nonlinear source and boundary damping terms, Glasgow Mathematical Journal 44 (2002), no. 3, 375-395.

[15] _ Global existence for the wave equation with nonlinear boundary damping and source terms, Journal of Differential Equations 186 (2002), no. 1, 259-298.

[16] Z. Yang and G. Chen, Global existence of solutions for quasi-linear wave equations with viscous damping, Journal of Mathematical Analysis and Applications 285 (2003), no. 2, 604-618.

Fuqin Sun: Department of Mathematics and Physics, Tianjin University of Technology and Education, Tianjin 300222, China

E-mail address: sfqwell@sina.com.cn

Mingxin Wang: Department of Mathematics, Southeast University, Nanjing 210096, China E-mail address: mxwang@seu.edu.cn 